\title{
SOURCES OF BEAM HALO IN THE NEXT LINEAR COLLIDER MAIN LINAC
}

\author{
P. Tenenbaum, T.O. Raubenheimer, M. Woodley, SLAC *
}

\section{Abstract}

One of the factors that limited the performance of the Stanford Linear Collider (SLC) was the number of particles per bunch with large betatron or energy amplitudes. We consider the equivalent problem for the Next Linear Collider (NLC) main X-band linacs. We evaluate the number of large-amplitude particles which can be expected due to scattering processes, wakefields, and magnet nonlinearities. We conclude that the number of particles in the beam halo from these sources can easily be accomodated by the planned post-linac collimation system.

\section{INTRODUCTION}

Modern colliders must balance the experimental requirements of high luminosity with low detector backgrounds. This struggle was particularly pronounced at the Stanford Linear Collider (SLC). Every reduction in the severity of the beam halo in the SLC was immediately followed by an improvement in the luminosity.

The Next Linear Collider (NLC) layout has been shaped by experience at the SLC: in particular, the NLC includes robust collimation of the beam halo immediately upstream and immediately downstream of the main X-band linacs [1]. The design of the collimation sections must include certain assumptions on the likely population of the beam halo which the collimators will encounter, the acceptable phase space of the halo from the point of view of detector backgrounds, and the efficiency with which the beam halo is attenuated.

The principal sources of beam halo in the NLC main linac itself are expected to be: scattering between beam particles in the core and thermal photons or residual gas atoms in the vacuum chamber; wakefield deflections of particles which trail the beam core; and quadrupole magnet nonlinearities which will drive particles to higher amplitudes. All of these sources will be considered. Note that in general, studies below are for the $500 \mathrm{GeV}$ per beam main linac configuration of 2000, and conform to the 2000 parameter sets.

\subsection{Halo Population and Transmission}

Studies of the NLC post-linac collimation system have indicated that the maximum halo population which can be accomodated is set by the muon flux generated when the $500 \mathrm{GeV}$ electrons are stopped [2]. The post-linac system can stop $10^{9}$ primary beam particles per linac pulse without exceeding the expected muon background limits of the

\footnotetext{
* Work supported by the U.S. Department of Energy, Contract DEAC03-76SF00515.
}

detector. This is $10^{-3}$ of the beam power, a halo population which is consistent with levels seen in later SLC runs [3].

The transmission of the post-linac collimation system is estimated to be $10^{-5}$, indicating that for every 100,000 primary-beam particles which encounters a collimator, only one is re-scattered back into the beam. The collimation efficiency is higher at lower beam energies, because the ratio of the multiple-coulomb scattering angle to the beam angular divergence is larger.

The halo transmission of the pre-linac collimation system has not been carefully reviewed. Based on the studies of the post-linac system, we assume that the pre-linac collimation system, at the $8 \mathrm{GeV}$ point in the NLC, will be at no larger than $10^{-3}$.

\subsection{Collimation Amplitude}

The maximum amplitude permitted near the detector is set by the requirement that synchrotron radiation emitted in the final doublet quadrupoles not strike the vertex detector. Because the doublet's SR has such a high critical energy, it cannot be masked; therefore, the particles which might generate such photons must be removed from the beam far upstream of the IP.

In the NLC design, the boundary between acceptable and unacceptable particle trajectories is a rectangle in $\left(x^{\prime *}, y^{\prime *}\right)$ space: the maximum allowable horizontal IP angle is 240 $\mu$ radians, the maximum vertical angle is $1 \mathrm{mrad}$. These limits translate to approximate collimator half-gaps of 220 $\mu \mathrm{m}(x)$ and $170 \mu \mathrm{m}(y)$. The energy aperture of the postlinac energy collimation system is $\pm 1 \%$.

\section{BEAM-GAS AND THERMAL PHOTON SCATTERING}

The scattering of particles from the beam core due to beam-gas and thermal photon interactions has become an increasingly important phenomenon in electron-positron accelerators. The LEP single-beam lifetime was, at high energies, dominated by such processes [4], and these were studied in considerable detail [5]. More recently the NLC beam delivery system configuration used in the design study [6] was analyzed to determine the potential for halo repopulation due to scattering of the beam core [7]. In this section, the formalism of [5] and [7] is used to estimate halo generation due to scattering in the main linac. The formalism must be modified for use in the linear accelerator environment to take adiabatic damping into account. The relevant modifications to the equations are discussed in detail elsewhere [8].

Throughout the following we assume that the residual 
gas in the accelerator is made up of $\mathrm{N}_{2}$, with atomic weight 14 , atomic number 7 , two atoms per molecule. We also assume that the thermal photon bath is made up of photons with $0.07 \mathrm{eV}$ (the mean energy of thermal photons at room temperature) with an isotropic distribution.

\subsection{Thermal Photons}

For $\mathrm{N}$ electrons in a vacuum chamber with length $L$, the distribution of energy loss $(\delta)$ from scattering off photons with number density $\bar{n}_{\gamma}$ is given by:

$$
\begin{aligned}
\frac{d N}{d \delta} & =\frac{N L \sigma_{0} \bar{n}_{\gamma}}{4 \pi} \int_{0}^{\alpha_{\max }} d \alpha \sin \alpha(1+\cos \alpha) \\
& \times \frac{1}{x}\left[\frac{1}{1-\delta}+1-\delta-4 r(1-r)\right]
\end{aligned}
$$

where $E$ is the electron energy, $\omega_{0}$ the photon energy, $\alpha$ the incidence angle, $\sigma_{0}$ the Thomson cross-section $\left(6.65 \times 10^{-29} \mathrm{~m}^{-2}\right) ; x \equiv 4 E \omega_{0} \cos ^{2}(\alpha / 2) / m_{e}^{2} c^{4} ;$ and $r \equiv \delta /(x(1-\delta))$. The upper limit of integration is the largest incident angle which can cause the energy loss $\delta$ : $\alpha_{\max } \approx 2 \cos ^{-1} \sqrt{\delta / 15.3 E[\mathrm{TeV}] \omega_{0}[\mathrm{eV}](1-\delta)}$. When properly applied to the NLC main linac, Equation 1 indicates that the total number of off-energy particles is 40 per bunch train of $10^{12}$.

The scattering angle required for a particle to exceed the betatron aperture of the post-linac collimators varies from 33 microradians at the $8 \mathrm{GeV}$ end of the main linac to 2.5 microradians at $500 \mathrm{GeV}$, but the RMS recoil angle of electrons from thermal-photon scattering is close to 137 nanoradians. Thus, we do not expect any measurable betatron halo from thermal-photon scattering.

\subsection{Beam-Gas Inelastic Scattering}

The cross-section for an inelastic beam-gas scattering event in which the beam loses an energy fraction greater than $\delta_{\min }$ is given by:

$$
\begin{array}{r}
\sigma_{\mathrm{BGB}}\left(\delta>\delta_{\min }\right)=\frac{A}{N_{A} X_{0}} \times \\
\left(-\frac{4}{3} \log \delta_{\min }-\frac{5}{6}+\frac{4}{3} \delta_{\min }-\frac{\delta_{\min }^{2}}{2}\right),
\end{array}
$$

where $A$ is the atomic mass (in $\mathrm{kg}$ per mole), $N_{A}$ Avogadro's number, and $X_{0}$ the radiation length (in $\mathrm{kg}$ per square meter) of the residual gas at STP. For a system pressure of $10 \mathrm{nTorr}$ of nitrogen gas, 1,700 off-energy particles are generated per bunch train.

The angular distribution of the photon emitted in inelastic beam gas scattering is approximately given by:

$$
f(\theta) d \Omega \approx \frac{0.392}{\gamma} \frac{\theta d \Omega}{\left(\theta^{2}+\gamma^{-2}\right)^{2}},
$$

where $\theta$ is the azimuthal angle of the photon and $\gamma$ is the electron relativistic factor. This expression allows an estimate of the number of electrons with a sufficient recoil angle to exceed the betatron collimation aperture: approximately 70 per bunch train at 10 nTorr pressure.

\subsection{Elastic Beam-Gas Scattering}

Coulomb scattering of electrons on residual gas atoms can only generate particles with a large betatron amplitude, not off-energy particles. The cross-section for scattering through an angle larger than $\theta_{\min }$ is given by:

$$
\begin{aligned}
\sigma_{\text {coulomb }}\left(\theta>\theta_{\min }\right) & \approx 6.5124 \times 10^{-8} \mathrm{bn} \\
& \times \frac{Z^{2}}{(E[\mathrm{GeV}])^{2}}\left[\frac{1}{1-\cos \theta_{\min }}\right] .
\end{aligned}
$$

At 10 nTorr pressure, Equation 4 indicates that 6,500 particles will scatter to amplitudes that exceed the collimation limits.

\subsection{Inelastic Scattering off Atomic Electrons}

The total cross-section for a beam electron to scatter off an atomic electron and suffer a fractional energy loss greater than $\delta_{\min }$ is given by:

$$
\sigma_{e^{-}, \delta_{\min }}=\frac{2 \pi r_{e}^{2} Z}{\gamma \delta_{\min }}
$$

The scattering angle in the laboratory frame is given by:

$$
\tan \theta=\frac{1}{\sqrt{2 \gamma}} \frac{2 \sqrt{\delta+\delta^{2}}}{1-2 \gamma}
$$

where $\delta$ is the actual energy lost by the electron. For 10 nTorr residual gas pressure, the number of off-energy electrons generated is less than one per bunch train, but the number of particles with an unacceptable betatron amplitude is approximately 1,900 per bunch train.

\section{TRANSVERSE WAKEFIELDS}

In the SLC, transverse wakefields were a significant source of beam halo: the linac beam included a longitudinal tail which was driven to large amplitudes by RF structure wakefields generated by the beam core. In the NLC, this is expected to be a much less significant source of beam halo for several reasons. First, the RMS beam-to-structure offset in the NLC will typically be small because the RF structures can be positioned by remote-controlled translation stages during beam operations, an option not available to the SLC. Second, the NLC main linac will operate closer to the "autophasing" condition, in which the transverse wakefields are cancelled by the chromaticity of the quadrupole lattice, than the SLC did [9]. Third, the NLC beam will be collimated in the longitudinal degree of freedom by a collimator at a point where both the dispersion and the $z-\delta$ correlation are large.

In simulation studies, a beam which has been collimated as described above was introduced into the NLC main linac with a vertical oscillation of $20 \sigma_{y}$. The influence of transverse wakefields drove approximately $10^{-5}$ of the beam out to the amplitude of the betatron collimators; this implies that for a huge oscillation up to $10^{7}$ primary beam particles per bunch train will be in the beam halo. Note that the 
luminosity dilution from such an oscillation is essentially total; the condition which produces $10^{7}$ halo particles per train does not approximate normal running.

\section{QUADRUPOLE NONLINEAR FIELDS}

In the SLC, the beam which was extracted from the damping rings was quite large compared to the magnet apertures in the transport lines that led back to the linac; this was believed to be a large contributor to the SLC beam's halo [10]. In the NLC, the beam size is a small fraction of the aperture in all magnets, and any halo from the damping ring and pre-linac beamlines will be reduced by the collimation system immediately upstream of the main linac. The beam which enters the main linac will therefore have three components: the beam core, which is approximately Gaussian and extremely intense; a more diffuse non-Gaussian halo, which will extend to the collimation aperture of the pre-linac collimators; and a very diffuse halo outside of the pre-linac collimation aperture, which is due to the fact that the pre-linac system has non-zero transmission of intercepted particles.

Although the beam core is so small that no realistic magnet imperfections could drive particles from the core into the halo, the "intermediate" halo which is within the aperture limits of the pre-linac system will be driven to larger amplitudes by nonlinear fields in the quads. The aperture of the pre-linac system must therefore be set such that the particles in the "intermediate halo" are not driven out of the aperture of the post-linac system.

The transport of the "intermediate halo" was simulated by combining an ensemble of particles with the nominal beam parameters with a rectangle of particles which lie at $95 \%$ of the acceptable aperture in $\left(x, x^{\prime}\right)$ or $\left(y, y^{\prime}\right)$ phase space. The program ELEGANT [11] is then used to transport the resulting particles through the linac.

The effects of 12-pole and 20-pole components in the linac quadrupoles are simulated. The multipole errors are based upon measurements of a prototype NLC magnet. The prototype's 12-pole and 20-pole fields were found to be $0.064 \%$ and $0.034 \%$ of the quadrupole field, respectively, at $60 \%$ of the aperture. The measured errors above are assigned to each quadrupole, to represent systematic errors due to construction. In addition, uniformly-distributed random 12- and 20-pole errors of up to $200 \%$ of the measured values above were added to each quad. Finally, the resulting multipole errors for each quad were multiplied by $10^{4}$.

Figure 1 shows the results of tracking. The upper two plots show the beam halo distribution at the end of the linac with no multipole errors, and the bottom two plots represent the results with the multipole errors described above. It is clear that, even for unreasonably large multipole errors, beam halo outside the post-linac collimation aperture due to multipoles can be eliminated by setting the pre-linac collimation apertures only marginally tighter.
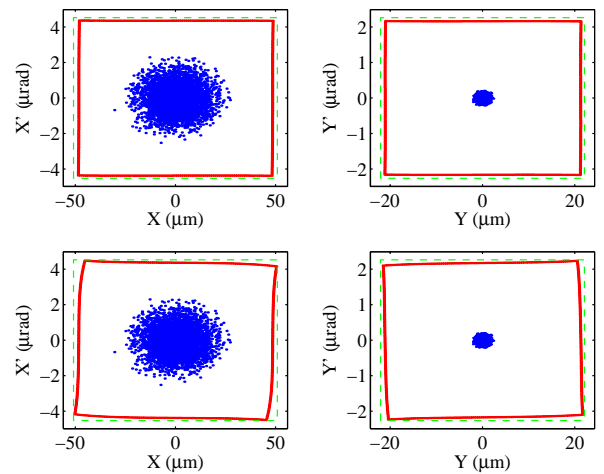

Figure 1: Beam core (blue) and boundary of halo (red) compared to detector acceptance (green): top figures are error-free, bottom figures include multipole errors described in the text.

\section{CONCLUSIONS}

We have considered scattering, wakefields, and multipole errors in the main linac as sources of beam halo. Scattering is expected to generate about $10^{4}$ halo particles per bunch train, of which $85 \%$ will impact the betatron collimators and $15 \%$ will impact the energy collimators. Wakefields could conceivably contribute up to $10^{7}$ particles per train, all of which would impact the betatron collimators, but the conditions required for such a large halo are far from the normal operating condition; under highluminosity conditions this source will be negligible. Multipole aberrations in the main linac will contribute nothing to the halo if the pre-linac collimators are set incrementally tighter than the post-linac collimators.

\section{REFERENCES}

[1] P. Tenenbaum et al, "Overview of Collimation at the Next Linear Collider," these proceedings.

[2] P. Tenenbaum et al, "Studies of Beam Optics and Scattering in the Next Linear Collider Postlinac Collimation System," Proceedings EPAC-2000 (2000).

[3] S. Hertzbach, "SR Fans in NLC FF," Proceedings $B D-I R$ 2000, http://accelerator.dl.ac.uk/ap/bdir2000/ proceedings.htm.

[4] H. Burkhardt, R. Kleiss, Proceedings EPAC-94 (1994).

[5] I. Reichel, CERN-Thesis-98-017 (1998).

[6] C. Adolphsen et al, SLAC-Report-474 (1996).

[7] I. Reichel et al, Proceedings ICAP-98 (1998).

[8] P. Tenenbaum, LCC-Note-0051, http://wwwproject.slac.stanford.edu/ lc/ilc/TechNotes/LCCNotes/ lcc_notes_index.htm.

[9] V.E. Balakin, A.V. Novokhatsky, V.P. Smirnov, Proceedings HEACC-83, 119 (1983).

[10] P. Emma, private communication.

[11] M. Borland, Advanced Photon Source LS-287 (2000). 